\title{
O.S.P.
}

L'orientation scolaire et professionnelle

$39 / 3 \mid 2010$

Travail et genre dans les médias

\section{Corroy (éd.). Les jeunes et les médias. Les raisons du succès}

Paris : Vuibert

Isabelle Olry-Louis

\section{(2) OpenEdition}

\section{Journals}

Édition électronique

URL : http://journals.openedition.org/osp/2878

DOI : $10.4000 /$ osp.2878

ISSN : 2104-3795

\section{Éditeur}

Institut national d'étude du travail et d'orientation professionnelle (INETOP)

Édition imprimée

Date de publication : 15 septembre 2010

Pagination : 422-423

ISSN : 0249-6739

Référence électronique

Isabelle Olry-Louis, «L. Corroy (éd.). Les jeunes et les médias. Les raisons du succès », L'orientation scolaire et professionnelle [En ligne], 39/3 | 2010, mis en ligne le 16 décembre 2010, consulté le 10 décembre 2020. URL : http://journals.openedition.org/osp/2878; DOI : https://doi.org/10.4000/osp. 2878

Ce document a été généré automatiquement le 10 décembre 2020.

(c) Tous droits réservés 


\section{Corroy (éd.). Les jeunes et les médias. Les raisons du succès}

Paris : Vuibert

Isabelle Olry-Louis

\section{RÉFÉRENCE}

Paris : Vuibert

1 Quels rapports les jeunes entretiennent-ils avec les médias d'aujourd'hui ? En quoi les usages qu'ils en font les distinguent-ils d'autres publics? Quels sont les supports médiatiques qui répondent le mieux à leurs attentes? Quelles raisons psychologiques, sociales et techniques peuvent être avancées pour expliquer ces modes de consommation propres aux adolescents? C'est à des questions de ce genre qu'une dizaine de chercheurs spécialistes des médias s'attèlent ici sous la houlette de Laurence Corroy, en passant en revue les supports médiatiques des plus traditionnels aux plus innovants.

Dans son introduction à l'ouvrage, l'auteur envisage trois raisons pour lesquelles les médias sont susceptibles d'intéresser les adolescents. Vecteurs d'informations, ils organisent et donnent sens aux évènements. C'est sans doute ce qui explique l'engouement du jeune public pour la presse gratuite ; c'est aussi la raison pour laquelle les forums de discussion sont largement investis, en ce qu'ils permettent d'obtenir des réponses aux questions que les jeunes se posent sur le monde et sur eux-mêmes. Réflecteurs d'attitudes, ils donnent aussi à voir des façons de se conduire via de nombreux témoignages. Ainsi en est-il des émissions interactives diffusées à la radio, des émissions de téléréalité ou encore des séries télévisuelles comme "Plus belle la vie». En offrant la possibilité de prendre la parole, ils constituent par ailleurs des supports de choix pour les lycéens avides de communiquer par SMS ou par blogs interposés. 
3 Le corps de l'ouvrage vise à déployer l'analyse à travers différents supports médiatiques en procédant à des études de cas approfondies. Organisé en trois parties, il examine successivement les médias papier (notamment la saga Harry Potter, les magazines destinés aux jeunes filles et les quotidiens gratuits), les émissions télévisuelles ("Plus belle la vie », " 24 heures chrono », ou encore " Loft Story ») et les nouveaux supports médiatiques (notamment les blogs, les SMS, les pratiques sonores en ligne et les forums sur internet).

4 On trouve, au fil des chapitres, de nombreuses informations chiffrées qui viennent éclairer les pratiques médiatiques. Ainsi, selon les plus récentes enquêtes de Médiamétrie, on apprend qu'avec les années 2000 un déclin durable et conséquent de l'audience presse s'est enclenché auprès des adolescents, et qu'une reprise, amorcée depuis 2 ans, semble cependant y avoir mis un terme. De façon générale, la lecture de la presse est devenue plus mobile, notamment grâce à la diffusion des quotidiens gratuits sur les lieux mêmes de transport. En matière de littérature jeunesse, Harry Potter a fait l'objet d'un succès médiatique sans nul autre pareil : 370 millions d'exemplaires vendus à travers le monde, traduits en 65 langues, avec 20 millions de volumes diffusés en France, records attribuables à sa dimension mythique et aux problématiques universelles sur l'ordre du monde et la possibilité pour chacun de s'y faire sa juste place - thématiques qui y sont développées. À côté des écrits, la télévision, bien que fortement concurrencée par Internet, reste le média familial par excellence, qui peut obtenir de fameux records d'audience. Le feuilleton « Plus belle la vie » diffusé à $20 \mathrm{~h} 20$ a ainsi obtenu $22,7 \%$ de parts de marché en 2007 , atteignant une audience de 35,2\% auprès des 11-14 ans et de 29,4\% auprès des 15-24 ans. Les séries télévisuelles font aussi réagir. Ainsi, en février 2008, plus de 195000 messages ont été postés par quelques 38000 membres sur le forum français de la série 24 heures chrono diffusée sur TF1 à partir de 2001. La radio constitue quant à elle un média d'accompagnement depuis fort longtemps : $88 \%$ des 13-24 ans l'écoutent chaque jour contre $70 \%$ des adultes, avec Skyrock comme leader pour cette tranche en réunissant 4 millions d'auditeurs. De son côté, Internet n'a cessé de gagner du terrain auprès des jeunes. Par ordre décroissant, les publics les plus connectés à Internet sont d'abord les 13-17 ans, suivis des 18-24 ans - en 2005, $80 \%$ d'entre eux étaient internautes, la plupart y consacrant plus d'une heure par jour, contre $50 \%$ des adultes. Environ $50 \%$ des 15-19 ans déclarent avoir créé un blog ou s'apprêtent à le faire et $80 \%$ des blogueurs ont moins de 24 ans.

5 On l'aura compris au vu de ces données, le jeune public se révèle être un consommateur assidu de médias, sous toutes les formes. Pour autant, le succès n'est pas toujours au rendez-vous. Quels sont alors les ressorts des programmes ou supports médiatiques dont s'empare la jeunesse? Pour répondre à une telle question, il convient de multiplier les approches: historiques, sémiotiques, techniques, psychosociales, langagières, la plupart des études de cas présentées dans l'ouvrage explorent plusieurs espaces d'analyse, ce qui en fait la richesse. Nous ne présenterons ici que deux exemples qui nous sont apparus particulièrement intéressants parce qu'ils se nourrissent de données empiriques ou théoriques très précises. Il s'agit du chapitre relatif aux émissions de télé-réalitéprésenté par Anne-Caroline Fiévet et du chapitre portant sur les forums adolescents rédigé par Florence Quinche.

6 Partant de l'idée que les émissions de télé-réalité visent à rassembler le plus grand nombre d'auditeurs en cherchant toutefois à assurer une proximité particulière entre les participants de ces émissions et les jeunes de la vie réelle, Anne-Caroline Fiévet se 
livre à une analyse lexicale du contenu conversationnel de certaines de ces émissions (du «Loft» à "Secret story ») pour savoir si les discours produits par les participants de ces émissions sont caractérisés par certains marqueurs langagiers. Une enquête complémentaire menée par entretien de groupe et par questionnaire lui permet d'examiner dans quelle mesure les jeunes - des étudiants en sciences du langage et de jeunes habitants des cités - se reconnaissent à travers l'emploi de ce vocabulaire. Ses conclusions plaident en faveur de discours à forte connotation "langue de jeunes " voire «langue de cité» (notamment dans «Loft story 2 ») auquel s'identifient modérément les sujets interrogés.

7 Florence Quinche examine les spécificités communicationnelles des forums pour adolescents, à la lumière des élaborations théoriques d'Erik Erickson sur le développement de l'identité à l'adolescence. Caractérisée par l'exploration des différentes personnalités pouvant être endossées, cette phase transitoire consiste à jouer avec l'image de soi en modifiant sa personnalité au gré des circonstances, des contextes et des désirs. Internet offre à cet égard un espace de liberté exceptionnel en proposant des sphères - celles des forums spécialisés - dans lesquels les adolescents peuvent virtuellement rencontrer d'autres adolescents partageant leurs goûts ou intérêts. Par le discours, ils peuvent alors donner un nouveau sens à ce qu'ils vivent et construire ainsi progressivement leur identité, se transformant à loisir en fonction des interlocuteurs. Selon l'auteur, le dialogue avec autrui apparaît central dans la construction de cette identité puisque "c'est en se racontant devant autrui [...] que l'on peut tester son identité, la manière dont elle est perçue par les autres, ce qu'elle provoque dans l'interlocution » (p. 158). Le changement d'interlocuteur et le jeu avec sa propre image - comme en témoignent les pseudonymes, images et autres avatars permettent à l'adolescent ainsi anonymé de tester sans incohérence de multiples postures idéologiques à partir d'arguments souvent construits ad hoc pour penser face aux autres, voire en opposition avec eux. De façon complémentaire, lire les autres amène à découvrir d'autres façons d'être, à se comparer à autrui, notamment sur les thèmes des sentiments et du corps et à se sentir ainsi affilié à une communauté. En somme, les forums constitueraient un média particulièrement approprié pour exprimer au moyen du langage ses fragilités tout autant que sa toute-puissance imaginaire et les confronter dialogiquement à l'altérité.

8 Cet ouvrage est incontestablement dans l'air du temps. Riche en études de terrain variées, il fournit une documentation abondante et des pistes de réflexions intéressantes sur des pratiques adolescentes contemporaines et sur les spécificités des médias qui prennent la jeunesse pour cible. On y trouvera aussi exprimées certaines postures critiques. Ainsi, Nicolas Becqueret remarque à propos de la radio combien le choix d'une chaîne peut faire l'objet d'une pression à l'uniformité dans certains milieux sociaux. Il observe par ailleurs que «les émissions s'adressant aux adolescents cherchent moins à les aider à passer de l'enfance à l'adolescence qu'à proposer et à permettre à des adultes (ceux qui les animent et les produisent) de parler et parfois de raisonner comme des adolescents» (p. 255), ce qui conduit l'auteur à s'interroger sur l'impact de ce type de discours écoutés quotidiennement à un âge où l'on est particulièrement influençable.

9 Ce livre passionnera assurément tous ceux qui s'intéressent aux médias. En développant une réflexion nourrie par un domaine d'expertise singulier - celui de l'information et de la communication - il dotera aussi les spécialistes de l'adolescence 
d'un regard nouveau qui leur permettra de mieux saisir les interactions que les collégiens, lycéens et étudiants entretiennent avec leur environnement via les médias. Complémentaire aux travaux qui abordent le développement de l'identité ou les relations familiales et amicales à l'adolescence, cet ouvrage constitue une synthèse de référence sur les pratiques médiatiques de la jeunesse. 